\title{
LIFETIMES OF THE MAGNETIC NETWORK ELEMENTS
}

\author{
YANG LIU AND HONGQI ZHANG \\ Beijing Astronomical Observatory, Beijing, China
}

\author{
HAIMIN WANG \\ Big Bear Solar Observatory, Caltech \\ Pasadena, CA 91125, U.S.A.
}

\begin{abstract}
Using the 155 hours of coordinated observing data of Huairou and Big Bear Solar Observatories, the lifetimes of network elements have been determined by different ways. The sample of network elements has been chosen on a strict criterion in order to reduce the bias. Two statistical methods with different considerations show a mean lifetime of elements of 49.20 hours and 54.92 hours, respectively. The least-square fit method provides an average lifetime of 55.84 hours.
\end{abstract}

\section{The OBSERVATIONAL DATA}

A series of magnetograms was obtained of a quiet region. The observing run spanned 155 hours with 6 night gaps of an average length of 5 hours from May 11, 1988 to May 17, 1988. The time resolution of the data was about 20 minutes. The target of this coordinated observing run was an enhanced network region (See Fig.1)

\section{THE LIFETIMES OF NETWORK ELEMENTS}

We selected all the magnetic network elements with flux greater than $10^{18} \mathrm{Mx}$ in this region, except an area in a radius of 15 " around the letter " $\mathrm{H}$ ", which is a concentration of enhanced network (See Fig. 1).

With this sample, we find that the decay of these network elements took place by three possible ways: (1) "Merging", which is not the common means that we usually mentioned, but here is the merging of the same polarity of the elements and the cancellation of the opposite polarity. (2) Breakup and (3) Unipolar decay.

From those we define that breaking or merging of a network element with another element means that the elements, die and new ones are born. Then, we use different ways to determine the lifetimes of the elements.

2.1. The Lifetimes of Network Elements Using the Counting Method 


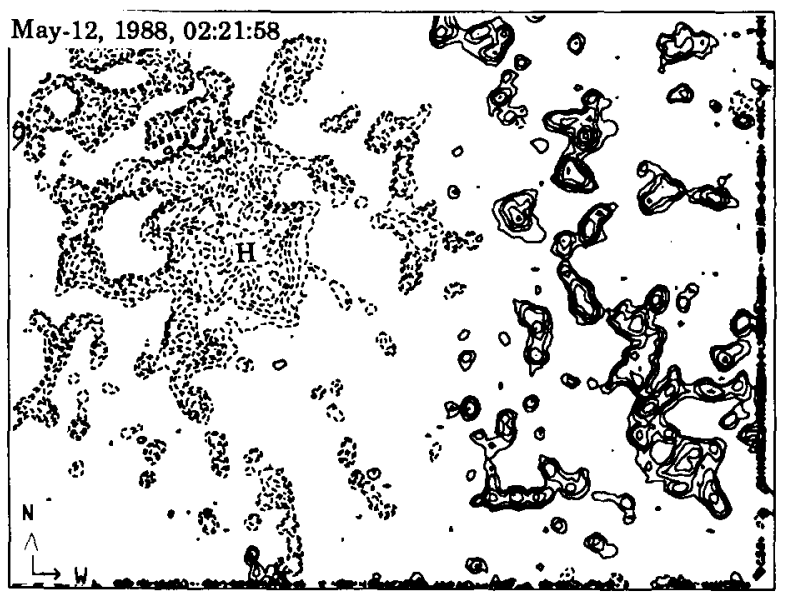

N :

$\mathbf{S}:$

Lev. : Gauss

2880.0

2560.0

2240.0

1920.0

1600.0

1280.0

960.0

640.0

320.0

160.0

80.0

40.0

20.0

Fig. 1: A solar quiet region with a 155 hours long-term coordinate observation. This is the longitudinal field on the photosphere.

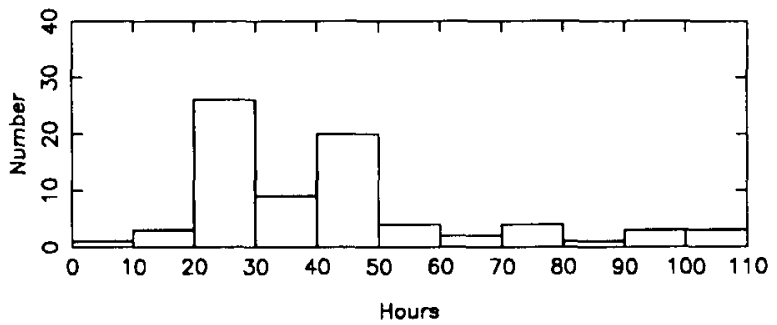

Fig. 2: The histogram shows the distribution of network elements lifetimes.

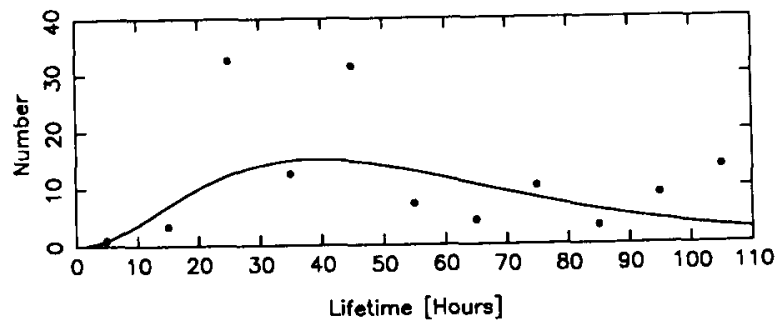

Fig. 3: The distribution of network elements lifetimes. The least-square fit gives a mean lifetime of network elements as 55.84 hours. The dots are the corrected observing data with the consideration of the weight factor $\mathrm{x}$. 
Table 1: The Lifetimes of Network Elements

\begin{tabular}{|c|c|c|c|}
\hline Sample & Categories & Number & $\begin{array}{c}\text { Lifetime } \\
\text { (Hours) }\end{array}$ \\
\hline Merging & $\begin{array}{l}\text { a: (Merging with the same polarity) } \\
\text { b: (Cancellation with the opposite } \\
\text { polarity) }\end{array}$ & 36 & 15 \\
\hline Breakup & & 25 & 50.55 \\
\hline$<1>:<2\rangle$ & & 2.04 & 54.92 \\
\hline
\end{tabular}

Table 2: The Lifetimes of Network Elements

\begin{tabular}{|c|c|c|}
\hline Sample & Number & Lifetime(Hours) \\
\hline Merging a: $_{\text {b: }}$ & 24 & 47.40 \\
\hline Breakup & 2 & 53.20 \\
\hline Average & 12 & 49.20 \\
\hline
\end{tabular}

Table 3: The Categories of Network Elements

\begin{tabular}{|c|c|c|c|c|c|}
\hline Categoriesidata & $12 \mathrm{th}$ & $13 \mathrm{~h}$ & $14 \mathrm{~h}$ & $15 \mathrm{~h}$ & Average \\
\hline$<1>\mathrm{a}:$ & 24 & 21 & 24 & 20 & 22.2 \\
\hline$<1>\mathrm{b}:$ & 5 & 1 & 2 & 6 & 3.5 \\
\hline$<2>$ & 13 & 12 & 10 & 12 & 11.8 \\
\hline$<1>:<2>$ & $2.2: 1$ & $1.8: 1$ & $2.6: 1$ & $2.1: 1$ & $2.2: 1$ \\
\hline
\end{tabular}

Remark: The category $\langle 1\rangle$ is the merging of network elements, in which $<a>$ means the merging of the same polarity elements and $\langle b\rangle$ is the cancellation of the opposite polarity elements. Category $<2>$ is the breakup. 
We only use those data which span about 123 hours in this 155 -hour coordinated observing. From these data, we were able to follow 76 network elements for their entire lifetime (See Fig. 2). Considering the weight factor of $\mathrm{x}=123 /(123+\mathrm{T})$, in which $\mathrm{T}$ is the lifetime of the element, we obtained an average lifetime of elements of 54.92 hours. The detailed results are listed in Table 1.

\subsection{The Lifetimes of Network Elements Using the Statistic Method}

Identifying and tracking every network element at the time 0000UT on May 12 as the beginning, we noted every interval between which the element we followed evolved and died. Two times of the average interval should be the average lifetime of the network elements. The average lifetime of all these elements is 49.2 hours (See Table 2).

Next, we express the relative number of elements as a function of their lifetimes as :

$$
\frac{f(T)=C \times T^{x}}{e^{\frac{T}{G}}-1.0}
$$

where $\mathrm{T}$ is the lifetime of elements, $\mathrm{C}, \mathrm{B}$ and $\mathrm{x}$ are constant. The least-square fit to this equation shows the distribution of network element lifetimes (See Fig. 3). The result is 55.84 hours.

\section{DISCUSSION}

With the coordinated observational data in the interval of 155 hours, the data we used span about 123 hours. Considering that the average lifetime of network elements is 55 hours with the consideration of the weight factor, the average number of network elements is 37 in the region, we can calculate the number of the network elements which undergo their whole evolution in the period of 123 hours as $\bar{N}=123 \times 37 / 55=82$, which fits the observing result of 76 elements.

Checking the properties of these 76 elements, we found that the number of "Merging" elements was about twice the number of "Breakup" elements (See Table 1). Classifying the network elements on May 12, 13, 14 and 15 with the same criterion, a similar phenomenon appeared (See Table 3 ). It is that the ratio of "Merging" network elements and the "Breaking" elements is about $2: 1$. Considering that the network elements in the category of "Merging" have almost the same average lifetime as those with the category of "Breaking up", the ratio of $2: 1$ had an obvious explanation: in average, one element usually breaks into two fragments and in the inverse process, two elements usually merge into a new element. If we consider the events of "Merging" and "Breakup" instead of numbers of elements, we find that the events of "Merging" are almost the same as the events of breaking into two fragments. In other words, the number of network magnetic elements is constant.

However, the lifetime study depends on the resolution of the observations. If we can see finer structure, the average lifetime may be reduced.

\section{CONCLUSIONS}

(1). The lifetime of the network magnetic elements is 55 hours.

(2). The number of network magnetic elements is constant. 